\title{
Special issue on control, communication, and complexity
}

\author{
Charalambos D. Charalambous • Pieter Collins • \\ Fritz Colonius
}

The subject of this special issue is the interplay between information transmission via digital communication channels and control of dynamical systems in real time. The problem of synthesizing and analyzing decentralized control and communication subsystems subject to delays and bandwidth constraints poses great theoretical and practical challenges which are the subject of current research. While the need for developing the theory was realized almost 20 years ago, the contours of promising approaches to the theory have only recently become visible. One line of research concerns the relations between control performance and communication performance, where the information patterns play a decisive role in the overall systems design. The development here is driven by applications such as control over the internet and wireless networks, and in general control over limited-bandwidth feedback. Communication complexity comes to the fore when one studies control system dynamics wherein multiple agents collaboratively provide inputs to a control system to achieve a common objective that no single agent could achieve alone. For hybrid systems containing continuous- and discrete-time elements, computability questions, in particular for reachability, are also of primary importance.

\section{D. Charalambous}

Department of Electrical and Computer Engineering, University of Cyprus,

75 Kallipoleos Avenue, 1678 Nicosia, Cyprus

e-mail: chadcha@ucy.ac.cy

P. Collins

Department of Knowledge Engineering, Maastricht University,

Postbus 616, 6200 MD Maastricht, The Netherlands

e-mail: pieter.collins@maastrichtuniversity.nl

\section{F. Colonius}

Institut für Mathematik, Universität Augsburg, Universitätsstrasse 14, 86159 Augsburg, Germany

e-mail: fritz.colonius@math.uni-augsburg.de 
This special issue collects five papers presenting different aspects of control, communication, and complexity.

The contribution by Paul Fuhrmann and Uwe Helmke deals with the control of large-scale networks of interconnected linear dynamical systems using the unifying framework of polynomial models. Their aim is to systematically study reachability and observability of finite networks of linear node systems. The models for the node systems are defined by first- or higher-order difference or differential equations. Using first-order representations for both the node systems and the interconnection dynamics, respectively, it is possible to apply standard reachability and observability tests for the closed-loop system. However, such an approach is problematic from a computational point of view, as it forces one to deal with matrices of large sizes. In fact, the Hautus criterion requires the checking of ranks of matrices whose format grows multiplicatively in the size of the network and the McMillan degrees of the subsystems. Thus, this approach becomes quickly inefficient for, e.g., medium-scale networks of highorder subsystems. Similarly, in the Kalman reachability or observability rank test, the simple linear interconnection structure of the network gets lost through the appearance of matrix powers. Thus, the effects of changes in the interconnection parameters are not clearly visible in first-order matrix reachability tests. Fuhrmann and Helmke show that using higher-order polynomial matrix fraction decompositions allows one to obtain very compact coprimeness conditions for reachability and observability. The main tool is a permanence principle for strict system equivalence based on early work of Rosenbrock and Pugh. They present a reformulation and extension of this principle to dynamic interconnections and deduce their main results on reachability and observability. This leads to new proofs for classical conditions for reachability and observability of series, parallel and feedback interconnections. Also, some new results for series or parallel connections of homogeneous networks are derived.

The contributions by Adriano Joao da Silva and by Rika Hagihara and Girish Nair extend the theory of topological feedback entropy that was introduced in a seminal paper by Nair, Evans, Mareels, and Moran (2004) in different directions. The classical notion of topological entropy in the theory of dynamical systems gives a measure for the total information produced by the system (it is a topological analogue of the Kolmogorov-Sinai entropy in the measure-theoretic context). In contrast, the goal of feedback entropy is to give universal bounds for the minimal information needed to perform diverse control tasks, in particular, for invariance of subsets of the state space. Thus, the notion of topological entropy had to be modified appropriately. From an engineering perspective, the operational significance of topological feedback entropy arises from the fact that it coincides with the smallest average bit rate between the plant and controller that allows set-invariance to be achieved. More precisely, suppose that an errorless digital channel with limited bit rate $R$ connects the plant sensor to the controller. Then a coder, controller and decoder that achieve set-invariance can be constructed if $R$ exceeds the topological feedback entropy of the plant and, conversely, $R$ must not be less than the topological feedback entropy. Thus, set-invariance is possible if and almost only if the digital channel can transport information faster than the plant generates it. A limitation of the above formulations of entropy for control systems is their restriction to plants with fully observed states and continuous dynamics. This makes them inapplicable when only a function of the state can be 
measured or the plant has discontinuities such as a quantized internal feedback loop. Rika Hagihara and Girish Nair generalize topological feedback entropy to continuous, partially observed systems, and to certain discontinuous, fully observed systems. They extend topological feedback entropy for both generalized situations and show that it coincides with the smallest average bit rate between the plant and controller that allows weak invariance to be achieved, thus giving these concepts operational relevance in communication-limited control. Though both generalizations involve open covers, the assumptions and techniques underlying them are significantly different. The questions of how to compute bounds on these notions and how to construct a unified notion of feedback entropy for discontinuous, partially observed systems are ongoing areas of research.

The paper by Adriano da Silva is based on an approach to invariance entropy for control systems using a metric on the state space, hence showing similarities to the Bowen-Dinaburg version of topological entropy in the theory of dynamical systems. He introduces and studies the notion of invariance entropy for continuous-time random control systems. Here, invariance is considered with respect to random compact sets. The main results provide upper and lower bounds for the invariance entropy of random pairs $(K, Q)$, where $K$ denotes the set of initial states and $Q$ the set of states in which the plant should stay. In particular, he shows that this entropy is finite, at least for a set of full measure. One important characteristic of these bounds is that they depend just on the random point $\omega$ and for the ergodic case they are constant. In the linear case, the entropy equals the sum of the positive Lyapunov exponents given by the Multiplicative Ergodic Theorem from the theory of random dynamical systems. Hence, the entropy for this case is an integrable function which is constant in the ergodic case.

The contribution by Rainer Blind and Frank Allgöwer analyzes the effects of the communication system and the interaction between control and communication for network control systems. The authors provide, under reasonable assumptions, a systematic analysis and comparison of the effects of time-triggered and event-based control over a shared communication system on the control performance. The control model assumed by Rainer Blind and Frank Allgöwer consists of $N$ plants, each described by a linear stochastic differential equation driven by the control process and an additive Brownian motion process, while the control actions applied are based on sampling the plant, and communicating the feedback information via a shared communication medium. The analysis on the interaction between communication and control, and its effects on control performance is done by considering different medium access control (MAC) protocols, which model the dependency between packet loss and delay on the network load and traffic. By taking the medium access into account the authors compare time-triggered and event-based control over the shared communication system. Specifically, for time-triggered control, where the event times are known à priori, they analyze time division multiple access (TDMA) and frequency division multiple access (FDMA) protocols, while for event-based control, where the events occur at random times, they analyze pure ALOHA, slotted ALOHA, a queueing system, and the Erlang loss model protocols. Under certain assumptions, it is shown that timetriggered control with either TDMA or FDMA outperforms event-based control with pure or slotted ALOHA, however the same conclusion cannot be made for eventbased control with a properly designed queueing system. Throughout the comparison 
analysis, it is also shown that the sampling strategy affects the performance not only directly but also indirectly via the loss and delay of the communication system, thereby demonstrating the effect of the communication system design on the control performance. Moreover, the authors demonstrate how the performance is also affected by the network load. The important conclusion is that when analyzing network control systems, it is important to take into account the effects of the interaction between the control and communication subsystems on the overall system performance. This contribution derives analytical models to describe such effects and provides a detailed analysis on the comparison between time-triggered and event-based control strategies for the different MAC mechanisms.

The paper by Azwirman Gusrialdi considers decentralized and distributed control with a particular emphasis on design of optimal communication topologies and an analysis of how performance is affected by permanent failure of communication links. The paper considers the case of interconnected heterogeneous linear time-invariant subsystems. A two-layer control architecture is proposed, comprising a local linear state-feedback controller for each subsystem, which is improved by allowing each local controller access to the states of subsystems with which it communicates directly. The first main result of the paper is the formulation of an optimal communication topology and distributed control law under costs on the network topology as a mixed-integer optimization problem. The second main result is a method for determining the effect of changes in the communication topology on the stability of the closed-loop system based on eigenvalue sensitivity analysis. The third main contribution is a detailed analysis of effective communication strategies for simple (line, ring and star-shaped) plant topologies. The theoretical contributions are supported with numerical results demonstrating the feasibility of the approach for medium-sized networks. These results suggest that in many cases, performance is determined by a few critical communication links.

As guest editors of this special issue we would like to thank the reviewers for their competent and constructive advice and the Editors-in-Chief of MCSS for the opportunity to compile this special issue on a fascinating area. 